\title{
A VARIATIONAL METHOD FOR FUNCTIONS OF BOUNDED BOUNDARY ROTATION
}

\author{
BY \\ BERNARD PINCHUK
}

Introduction. In this paper we present a variational method for functions of bounded boundary rotation and solve certain general extremal problems for these functions. The variational method is based upon a general method of G. M. Goluzin [1], and has previously been used by the present author to solve extremal problems for several classes of univalent functions ([7] and [8]). Once the variation formulas are derived, the details of solving the extremal problems are very similar to those in [7] and [8].

Theorem 1 here has been obtained independently by Professor J. Pfaltzgraff, who used essentially the same methods. I wish to thank Professor Pfaltzgraff for providing me with his unpublished work.

Those functions of bounded boundary rotation which are also univalent form a subclass which has recently been studied by M. M. Schiffer and O. Tammi [9]. They use a different (though closely related) variational method. We shall compare their results with ours.

1. Preliminaries. Let $V_{k}$ denote the class of normalized analytic functions $f(z)=z+a_{2} z^{2}+\cdots$ in the open unit disc $D=\{z:|z|<1\}$ which satisfy the condition $f^{\prime}(z) \neq 0$ for all $z$ in $D$, and which map $D$ onto a domain with boundary rotation bounded by $k \pi$.

V. Paatero [6] has shown that $f(z) \in V_{k}$ iff

$$
f^{\prime}(z)=\exp \left(\int_{0}^{2 \pi}-\log \left(1-z e^{-i \theta}\right) d \psi(\theta)\right)
$$

where $\psi(\theta)$ is a real-valued function of bounded variation with

$$
\int_{0}^{2 \pi} d \psi(\theta)=2 \text { and } \int_{0}^{2 \pi}|d \psi(\theta)| \leqq k .
$$

The geometric interpretation is as follows (see [5]): Let $f(z)$ map $D$ onto a domain $G$. If $G$ is a schlicht domain with a continuously differentiable boundary curve, let $\pi \psi(\theta)$ denote the angle of the tangent vector at the point $f\left(e^{i \theta}\right)$ to the boundary curve with respect to the positive real axis. The boundary rotation of $G$ is equal to $\pi \int_{0}^{2 \pi}|d \psi(\theta)|$. If $G$ does not have a sufficiently smooth boundary curve,

Received by the editors February 26, 1968 and, in revised form, April 1, 1968. 
the boundary rotation is defined by a limiting process. Finally, if $G$ is a nonschlicht domain without interior branch points, the boundary rotation is similarly defined.

We define $S_{k}$ to be the subclass of $V_{k}$ consisting of the univalent functions in $V_{k}$.

2. Univalent functions. Clearly, $f(z) \in V_{2}$ iff $f(z)$ is a normalized univalent function mapping $D$ onto a convex domain. Furthermore, V. Paatero [6] has shown that for $2 \leqq k \leqq 4$ the classes $V_{k}$ consist entirely of univalent functions. We strengthen this assertion by showing:

Proposition 1. For $2 \leqq k \leqq 4$, the classes $V_{k}$ consist entirely of close-to-convex functions.

Proof. The proof of this proposition consists of stating an appropriate definition of close-to-convex functions. We choose the geometric interpretation originally given by Kaplan [3, p. 177]. Let $f(z)$ be analytic in $D$ and continuously differentiable for $z=e^{i \theta}, 0 \leqq \theta \leqq 2 \pi . f(z)$ is close-to-convex if the angle of the tangent vector to the boundary curve of the image of $D$ under $f(z)$ with respect to the positive real axis either increases as $\theta$ increases or else decreases in such a manner that it never drops to a value $\pi$ radians below a previous value. If $f\left(e^{i \theta}\right)$ is not sufficiently smooth, we appeal to the suitable limiting process (see also [2, p. 391]).

Now, if $f(z) \in V_{k}, 2 \leqq k \leqq 4$, it clearly satisfies the above conditions and is closeto-convex. Since every close-to-convex function is univalent, see [3], the observation of Paatero is contained here.

3. Variations for $V_{k}$. G. M. Goluzin [1] derived variational formulas for classes $E_{g}$ of analytic functions in $D$ defined by the condition: $f(z) \in E_{g}$ iff

$$
f(z)=\int_{0}^{2 \pi} g(z, \theta) d \psi(\theta)
$$

where $g(z, \theta)$ is a given function, analytic in $D$ for $0 \leqq \theta \leqq 2 \pi$, and $\psi(\theta)$ runs through all nondecreasing functions on $0 \leqq \theta \leqq 2 \pi$ subject to the condition $\int_{0}^{2 \pi} d \psi(\theta)=A$.

Goluzin's variations consist of appropriately varying the function $\psi(\theta)$ so as to preserve its monotonicity and total variation.

For a given function $f(z) \in E_{g}$ having the representation (2) Goluzin derived the following variational formulas:

$$
f_{*}(z)=f(z)+\lambda \int_{\theta_{1}}^{\theta_{2}} \frac{\partial g(z, \theta)}{\partial \theta}|\psi(\theta)-c| d \theta
$$

where $\theta_{1}$ and $\theta_{2}$ are arbitrary but fixed with $0 \leqq \theta_{1}<\theta_{2} \leqq 2 \pi, \lambda$ varies in the interval $-1 \leqq \lambda \leqq 1$, and $c$ is real number independent of $\lambda$ and $\theta$.

$$
f_{* *}(z)=f(z)+\lambda\left(g\left(z, \theta_{1}\right)-g\left(z, \theta_{2}\right)\right)
$$

where $\theta_{1}$ and $\theta_{2}$ with $0 \leqq \theta_{1}<\theta_{2} \leqq 2 \pi$ are jump points for $\psi(\theta)$, and $\lambda$ varies in the interval $-a<\lambda<a$, where $a>0$ is sufficiently small. 
To apply these variational formulas to $V_{k}$, we express the function $\psi(\theta)$ appearing in (1) as the difference of two increasing functions, $\psi(\theta)=\alpha(\theta)-\beta(\theta)$. Since

$$
\int_{0}^{2 \pi} d \psi(\theta)=\int_{0}^{2 \pi} d \alpha(\theta)-\int_{0}^{2 \pi} d \beta(\theta)=2
$$

and

$$
\int_{0}^{2 \pi}|d \psi(\theta)|=\int_{0}^{2 \pi} d \alpha(\theta)+\int_{0}^{2 \pi} d \beta(\theta) \leqq k,
$$

we conclude that

$$
\int_{0}^{2 \pi} d \alpha(\theta) \leqq \frac{k}{2}+1
$$

and

$$
\int_{0}^{2 \pi} d \beta(\theta) \leqq \frac{k}{2}-1
$$

The representation (1) for $V_{k}$ can now be rewritten as

$$
f^{\prime}(z)=\exp \left(\int_{0}^{2 \pi} \log \left(1-z e^{-i \theta}\right) d \beta(\theta)-\int_{0}^{2 \pi} \log \left(1-z e^{-i \theta}\right) d \alpha(\theta)\right)
$$

where $\alpha(\theta)$ and $\beta(\theta)$ are nondecreasing functions on $[0,2 \pi]$ satisfying (5) and (6) respectively.

(7) can be written as

$$
f^{\prime}(z)=\exp (H(z)-G(z))
$$

where

and

$$
H(z)=\int_{0}^{2 \pi} \log \left(1-z e^{-i \theta}\right) d \beta(\theta)
$$

$$
G(z)=\int_{0}^{2 \pi} \log \left(1-z e^{-i \theta}\right) d \alpha(\theta) .
$$

As $\alpha(\theta)$ and $\beta(\theta)$ range over all nondecreasing functions satisfying (5) and (6) respectively, we obtain two classes of the form $E_{g}$ described above. We apply the Goluzin formulas to these classes.

Varying $H(z)$ we obtain for $V_{k}$,

$$
\begin{aligned}
f_{*}^{\prime}(z) & =\exp \left(H_{*}(z)-G(z)\right)=\exp \left(H(z)-G(z)+\lambda \int_{\theta_{1}}^{\theta_{2}} \frac{i z e^{-i \theta}}{1-z e^{-i \theta}}|\beta(\theta)-c| d \theta\right) \\
& =f^{\prime}(z) \cdot \exp \left(\lambda \int_{\theta_{1}}^{\theta_{2}} \frac{i z e^{-i \theta}}{1-z e^{-i \theta}}|\beta(\theta)-c| d \theta\right) .
\end{aligned}
$$

Expanding the exponential up to first order terms in $\lambda$ we have,

$$
f_{*}^{\prime}(z)=f^{\prime}(z)+\lambda \int_{\theta_{1}}^{\theta_{2}} f^{\prime}(z) \frac{i z e^{-i \theta}}{1-z e^{-i \theta}}|\beta(\theta)-c| d \theta+O\left(\lambda^{2}\right) .
$$


Here, $O\left(\lambda^{2}\right)$ is an analytic function of $z$, and the error is uniform in each disc $|z| \leqq r<1$.

Varying $G(z)$ we obtain the same formula with $\beta(\theta)$ replaced by $\alpha(\theta)$.

If $\theta_{1}$ and $\theta_{2}$ are jump points for $\alpha(\theta)$ or $\beta(\theta)$ we have the following variational formula for $V_{k}$ :

$$
f_{* *}^{\prime}(z)=f^{\prime}(z)+\lambda f^{\prime}(z)\left(\log \left(1-z e^{-i \theta_{1}}\right)-\log \left(1-z e^{-i \theta_{2}}\right)\right)+O\left(\lambda^{2}\right) .
$$

It should be remarked at this point that the formulas (8) and (9) are identical in form to the variational formulas derived in [8] for convex functions of order $\alpha$. It is for this reason that, as mentioned in the introduction, the details to the solution of extremal problems for $V_{k}$ will be very similar to those in [8].

4. A general extremal problem. The solution of extremal problems for $V_{k}$ is equivalent to finding the functions $\alpha(\theta)$ and $\beta(\theta)$ which appear in the representation (7) for the extremal function. We begin with a general extremal problem for $V_{k}$.

THeOREM 1. Let $\zeta \neq 0$ be a given point in $D$, and let $F\left(X_{1}, X_{2}, \ldots, X_{n+1}\right)$ be analytic in a neighborhood of each point $F\left(f^{\prime}(\zeta), f^{\prime \prime}(\zeta), \ldots, f^{(n)}(\zeta), \zeta\right), f(z) \in V_{k}$. Then the functional

$$
J\left(f^{\prime}\right)=\operatorname{Re} F\left(f^{\prime}(\zeta), f^{\prime \prime}(\zeta), \ldots, f^{(n)}(\zeta), \zeta\right)
$$

attains its maximum (or minimum) in $V_{k}$ only for a function of the form

$$
f^{\prime}(z)=\prod_{j=1}^{M}\left(1-\varepsilon_{j} z\right)^{\beta_{j}} / \prod_{j=1}^{N}\left(1-e_{j} z\right)^{\alpha_{j}}
$$

where $M<n, N \leqq n,\left|\varepsilon_{j}\right|=\left|e_{j}\right|=1$ and $\sum_{j=1}^{M} \beta_{j} \leqq k / 2-1$ and $\sum_{j=1}^{N} \alpha_{j} \leqq k / 2+1$.

Proof. The compactness of $V_{k}$ assures the existence of a function $f(z) \in V_{k}$ which maximizes (or minimizes) $J\left(f^{\prime}\right)$. Let $f(z)$ with

$$
f^{\prime}(z)=\exp \int_{0}^{2 \pi} \log \left(1-z e^{-i \theta}\right)(d \beta(\theta)-d \alpha(\theta))
$$

be such a function. For the functions $f_{*}^{\prime}(z)$ defined by (8) we have

(11) $J\left(f_{*}^{\prime}\right)=J\left(f^{\prime}\right)+\lambda \int_{\theta_{1}}^{\theta_{2}} \operatorname{Re} \sum_{j=1}^{n} d_{j}\left[i \zeta f^{\prime}(\zeta)\left(e^{i \theta}-\zeta\right)^{-1}\right]^{(j-1)}|\beta(\theta)-c| d \theta+O\left(\lambda^{2}\right)$

where $d_{j}=\partial F\left(X_{1}, \ldots, X_{n+1}\right) / \partial X_{j}$ evaluated at the extreme point

$$
\left(f^{\prime}(\zeta), \ldots, f^{(n)}(\zeta), \zeta\right)
$$

(Here, $f^{(0)}(z)=f(z)$.)

The extremal property of $f(z)$ implies that

$$
\int_{\theta_{1}}^{\theta_{2}} Q(\theta)|\beta(\theta)-c| d \theta=0
$$


where

$$
Q(\theta)=\operatorname{Re} \sum_{j=1}^{n} d_{j}\left[i \zeta f^{\prime}(\zeta)\left(e^{i \theta}-\zeta\right)^{-1}\right]^{(j-1)}
$$

$V_{k}$ is a rotation invariant family, and a theorem of Kirwan [4] assures us that at least one $d_{j} \neq 0$.

If the continuous function $Q(\theta)$ does not vanish in the interval $\left(\theta_{1}, \theta_{2}\right),(12)$ implies that $\beta(\theta)=c$ throughout that interval. Now, $Q(\theta)=0$ is equivalent to an equation of degree at most $2 n$ in $e^{i \theta}$. We therefore conclude that $\psi(\theta)$ is a step function with at most $2 n$ jump points and furthermore, these jumps can only occur at the zeros of $Q(\theta)$. Let these points be $\theta_{1}, \ldots, \theta_{2 n}$, with $\theta_{1}<\theta_{2}<\cdots<\theta_{2 n}$.

We now construct the functions $f_{* *}^{\prime}(z)$ defined by (9). For these functions we have

$$
\begin{aligned}
J\left(f_{* *}^{\prime}\right)= & J\left(f^{\prime}\right) \\
& +\lambda \operatorname{Re} \sum_{j=1}^{n} d_{j}\left[f^{\prime}(\zeta)\left(\log \left(1-\zeta e^{-i \theta_{1}}\right)-\log \left(1-\zeta e^{-i \theta_{2}}\right)\right]^{(j-1)}+O\left(\lambda^{2}\right) .\right.
\end{aligned}
$$

The extremal property of $f^{\prime}(z)$ implies that the coefficient of $\lambda$ in (13) must vanish. Thus, the function

$$
R(\theta)=\operatorname{Re} \sum_{j=1}^{n} d_{j}\left[f^{\prime}(\zeta) \log \left(1-\zeta e^{-i \theta}\right)\right]^{(j-1)}
$$

has the same value at $\theta_{1}$ and $\theta_{2}$. The Mean Value Theorem implies that $R^{\prime}(\theta)$ must vanish at some point $\theta^{\prime}$, with $\theta_{1}<\theta^{\prime}<\theta_{2}$. But $R^{\prime}(\theta)=Q(\theta)$ and this is a contradiction.

We conclude that $\beta(\theta)$ could not have jump points at each of the $2 n$ roots of $Q(\theta)$, but at most at every other root. Thus, $\beta(\theta)$ is a step function with at most $n$ jump points.

A similar procedure with $\beta(\theta)$ replaced by $\alpha(\theta)$ in (8) and (9) yields the conclusion that $\alpha(\theta)$ is a step function having at most $n$ jump points. Evaluating (7) with these $\alpha(\theta)$ and $\beta(\theta)$ we arrive at $(10)$ and the theorem is proved.

For the class $V_{k}^{\prime}$ of functions with boundary rotation equal to $k \pi$, the conclusion of Theorem 1 is strengthened to $\sum \alpha_{j}=k / 2+1$ and $\sum \beta_{j}=k / 2-1$. Furthermore, as we shall see in the next section, for certain specific extremal problems in $V_{k}$ we also conclude $\sum \alpha_{j}=k / 2+1$ and $\sum \beta_{j}=k / 2-1$.

5. Distortion and rotation theorems. We begin with the known distortion theorem for $V_{k}$ [5].

Corollary 1. Let $f(z) \in V_{k}$. Then,

$$
\frac{(1-|z|)^{k / 2-1}}{(1+|z|)^{k / 2+1}} \leqq\left|f^{\prime}(z)\right| \leqq \frac{(1+|z|)^{k / 2-1}}{(1-|z|)^{k / 2+1}} .
$$

These bounds are sharp and are attained by a function of the form (10) with $M=N=1$. 
Proof. Set $J\left(f^{\prime}\right)=\left|f^{\prime}(\zeta)\right|$ in Theorem 1.

CoROllary 2. Let $f(z) \in V_{k}$. Then for all $z \in D$,

$$
\left|\arg f^{\prime}(z)\right| \leqq k \sin ^{-1}|z|
$$

where $\sin ^{-1} 0=0$.

This bound is sharp and is attained by a function of the form (10) with $M=N=1$.

Proof. Set $J\left(f^{\prime}\right)=\operatorname{Re} \pm i \log f^{\prime}(\zeta)$ in Theorem 1, and recall that

$$
\left|\arg \left(1-r e^{i \theta}\right)\right| \leqq \sin ^{-1} r .
$$

It is interesting to observe that this is the same sharp bound on $\left|\arg f^{\prime}(z)\right|$ obtained by Schiffer and Tammi [9, p. 143], for $S_{k}$.

If the functional $J(f)$ has the form $J(f)=\operatorname{Re} F\left(\log f^{\prime}(\zeta)\right)$ we can strengthen the result of Theorem 1. In this case, the extremal function must be of the form

$$
f^{\prime}(z)=(1-\varepsilon z)^{k / 2-1} /(1-e z)^{k / 2+1}
$$

with $|\varepsilon|=|e|=1$.

\section{A general coefficient theorem.}

THEOREM 2. Let $F\left(X_{2}, \ldots, X_{n}\right)$ be analytic in $C^{n-1}$. Then the functional

$$
C(f)=\operatorname{Re} F\left(a_{2}, \ldots, a_{n}\right)
$$

where $f(z)=z+a_{2} z^{2}+\cdots$, attains its maximum (or minimum) in $V_{k}$ only for a function of the form

$$
f^{\prime}(z)=\prod_{j=1}^{N}\left(1-\varepsilon_{j} z\right)^{\beta_{j}} / \prod_{j=1}^{M}\left(1-e_{j} z\right)^{\alpha_{j}}
$$

where $N \leqq n-1, M \leqq n-1,\left|\varepsilon_{j}\right|=\left|e_{j}\right|=1$, and $\sum_{j=1}^{N} \beta_{j} \leqq k / 2-1$ and $\sum_{j=1}^{M} \alpha_{j} \leqq k / 2+1$.

Proof. The proof is quite similar to the proof of Theorem 1 and we merely sketch it.

Let $f(z)=z+\sum_{n=2}^{\infty} a_{n} z^{n}$ be extremal for the problem under consideration with

$$
f^{\prime}(z)=\exp \int_{0}^{2 \pi} \log \left(1-z e^{-i \theta}\right)(d \beta(\theta)-d \alpha(\theta)) .
$$

For the functions $f_{*}^{\prime}(z)$ given by $(8)$ we have

$$
f_{*}(z)=z+\sum_{n=2}^{\infty}\left(a_{n}+\lambda \int_{\theta_{1}}^{\theta_{2}} \delta a_{n}|\beta(\theta)-c| d \theta\right) z^{n}+O\left(\lambda^{2}\right)
$$

with

$$
\delta a_{n}=\frac{i}{n} \sum_{j=0}^{n-2}(j+1) a_{j+1} \exp (-i(n-j-1) \theta), \quad\left(a_{1}=1\right) .
$$


Computing $C\left(f_{*}\right)$ and exploiting the extremal property of $f(z)$ we arrive at the condition

$$
\int_{\theta_{1}}^{\theta_{2}} \operatorname{Re} \sum_{j=2}^{n} d_{j} \delta a_{j}|\beta(\theta)-c| d \theta=0 .
$$

The continuous function $Q(\theta)=\operatorname{Re} \sum_{j=2}^{n} d_{j} \delta a_{j}$ has at most $2 n-2$ roots with respect to $\theta$ in $[0,2 \pi]$. Thus, $\beta(\theta)$ is a step function with at most $2 n-2$ jump points. Now, constructing $C\left(f_{* *}\right)$ we can prove, using the same reasoning as in the proof of Theorem 1 , that $\beta(\theta)$ and $\alpha(\theta)$ are step functions having at most $n-1$ jump points, and the theorem is proved.

Setting $C(f)=\left|a_{2}\right|$, we conclude the known result (see e.g., [5]) that the function maximizing $\left|a_{2}\right|$ over $V_{k}$ is a function of the form (14) with $N=M=1, \beta_{1}=k / 2-1$, $\alpha_{1}=k / 2+1$.

For the problem of maximizing $\left|a_{n}\right|$ over $S_{k}$, Schiffer and Tammi [9, p. 137], have found the extremal function to be a mapping of $D$ onto a polygon with $N$ corners and $N \leqq 2(n-2)$. This agrees with the conclusion of Theorem 2 . The expression for the extremal function in Theorem 2, (14), gives a bit more information about the structure of the extremal function.

\section{REFERENCES}

1. G. M. Goluzin, On a variational method in the theory of analytic functions, Amer. Math. Soc. Transl. (2) 18 (1961), 1-14.

2. W. K. Hayman, Coefficient problems for univalent functions and related function classes, J. London Math. Soc. 40 (1965), 385-406.

3. W. Kaplan, Close-to-convex schlicht functions, Michigan Math. J. 1 (1952), 169-185.

4. W. E. Kirwan, A note on extremal problems for certain classes of analytic functions, Proc. Amer. Math. Soc. 17 (1966), 1028-1030.

5. $\mathrm{O}$. Lehto, On the distortion of conformal mappings with bounded boundary rotation, Ann. Acad. Sci. Fenn. Ser. AI 124 (1952).

6. V. Paatero, Ubber die konforme Abbildungen von Gebieten deren Ränder von beschränkter Drehung sind, Ann. Acad. Sci. Fenn. Ser. A 33 (1931), No. 9.

7. B. Pinchuk, Extremal problems in the class of close-to-convex functions, Trans. Amer. Math. Soc. 129 (1967), 466-478.

8. - On starlike and convex functions of order $\alpha$, Duke Math. J. 35 (1968).

9. M. M. Schiffer and O. Tammi, A method of variation for functions with bounded boundary rotation, J. Analyse Math. 17 (1966), 109-144.

Princeton University,

Princeton, NeW Jersey 